\title{
Laminar forced convection at low Péclet number
}

\begin{abstract}
A.S. Jones
This work is concerned with the forced convection of heat in a circular tube. The fluid flow is assumed to be laminar Poiseuille flow, and the physical parameters; viscosity, density, conductivity; are assumed to be independent of temperature changes. Viscous dissipation terms are also ignored, and there are no heat sources in the fluid. The problem is treated for the case of a step change in the wall temperature, and the eigenvalues have been obtained as an expansion in powers of the Péclet number for the smaller values, and in an asymptotic form for the larger values. The temperature distribution in the fluid in the neighbourhood of the temperature jump has been calculated for two values of the Péclet number, as have the Nusselt numbers.
\end{abstract}

\section{Introduction}

This problem has received a great deal of attention in the case where the Péclet number is large, but not when it is small. Singh [5] calculated the negative eigenvalues for $P \hat{e}=1$, while Abramowitz, Cahill and Wade [1] calculated the eigenvalues when $P \dot{e}=\frac{1}{2}$ together with the coefficients in the expansion of the solution. These coefficients, however, were calculated on the dubious assumption that there is no preheating of the fluid. Millsaps and Pohlhausen [3] calculated the eigenvalues and thermal properties for $P \dot{e}=1$, but they too assumed no preheating of the fluid.

In the two-dimensional case, Agrawal [2] included the effects of

Received 13 September 1971. 
preheating and calculated eigenvalues, Nusselt numbers and temperature profiles for $P \hat{e}=1$. One criticism is that the method adopted for matching the temperature distribution upstream and downstream is unnecessarily complicated when compared with the Laplace transform method outlined below.

\section{Governing equations and their solution}

For the case of Poiseuille flow in a circular tube of radius $a$, the axi-symmetric conduction convection equation is

$$
\frac{\partial^{2} T}{\partial r^{2}}+\frac{1}{r} \frac{\partial T}{\partial r}+\frac{\partial^{2} T}{\partial x^{2}}=\frac{2 u_{m} \rho^{*} c}{\kappa}\left(1-\frac{r^{2}}{a^{2}}\right) \frac{\partial T}{\partial x},
$$

where

$T$ is the fluid temperature,

$u_{m}$ is the mean fluid velocity,

$\rho^{*}$ is the fluid density,

$c_{v}$ is the specific heat of the fluid, and

$\kappa$ is the thermal conductivity of the fluid.

The variables $r, x$ are the usual radial and axial variables in cylindrical polar coordinates, and the angular variable disappears because of the symmetry of the problem.

The boundary conditions imposed on equation 2.1 are

$$
\begin{aligned}
& T=T_{0} ; r=a, \quad x<0 \\
& T=T_{1} ; r=a, \quad x>0 \\
& T \rightarrow T_{0} ; x \rightarrow-\infty, \quad x<a \\
& T \rightarrow T_{1} ; x \rightarrow+\infty, r<a \text {. }
\end{aligned}
$$

The equation and boundary conditions are made non-dimensional by putting

$$
\rho=r / a, \quad \xi=x / \alpha, \quad \theta=\left(T-T_{0}\right) /\left(T_{1}-T_{0}\right),
$$

giving

$$
\frac{\partial^{2} \theta}{\partial \rho^{2}}+\frac{1}{\rho} \frac{\partial \theta}{\partial \rho}+\frac{\partial^{2} \theta}{\partial \xi^{2}}=2 P \bar{e}\left(1-\rho^{2}\right) \frac{\partial \theta}{\partial \xi}
$$

with the boundary conditions 
$(2.4)$

$$
\begin{array}{lll}
\theta=0 ; & \rho=1, & \xi<0 \\
\theta=1 ; & \rho=1, & \xi>0 \\
\theta \rightarrow 0 ; & \xi \rightarrow-\infty, & \rho<1 \\
\theta \rightarrow 1 ; & \xi \rightarrow+\infty, & \rho<1,
\end{array}
$$

where $P \dot{e}$ is the non-dimensional Péclet number ${ }^{(*)} u_{m}{ }^{* *} c_{v} a / k$.

The formal solution of equation (2.3) is obtained by using the double-sided Laplace transform.

Writing

$$
\bar{\theta}(p, \rho)=\int_{-\infty}^{\infty} e^{-p \xi} \theta(\xi, \rho) d \xi
$$

we obtain

$$
\frac{\partial^{2} \bar{\theta}}{\partial p^{2}}+\frac{1}{\rho} \frac{\partial \bar{\theta}}{\partial \rho}+\left(p^{2}-2 p P \bar{e}\left(1-\rho^{2}\right)\right) \bar{\theta}=0
$$

with boundary conditions

$$
\bar{\theta}(p, 1)=1 / p \text {. }
$$

Equation (2.5) is a second order linear ordinary differential equation for $\bar{\theta}$ with variable $\rho$ and parameters $p$, Pé . The equation has a regular singular point at $\rho=0$ with indices 0,0 . Consequently there is one independent solution as a power series in $\rho$, all other solutions having a logarithmic singularity at $\rho=0$. Since the temperature will remain finite at $\rho=0$, we can determine a unique solution $f(\rho ; p, P e ́)$ of equation (2.5) from the condition $f(0 ; p, P e ́)=1$. The required solution for $\bar{\theta}$, satisfying the boundary conditions is then

$$
\bar{\theta}=\frac{1}{p} \frac{f(\rho ; p, P e ́)}{f(1 ; p, P e ́)} \text {. }
$$

The function $\theta$ is recovered by using the inverse Laplace transform

$$
\theta(\xi, \rho)=\frac{1}{2 \pi i} \int_{c-i \infty}^{c+i \infty} e^{\xi p \bar{\theta}}(p, \rho) d p,
$$

where

(*) Some authors define the Péclet number to be twice this value. 


$$
0<c<\text { the first positive pole of } \bar{\theta} \text {. }
$$

This gives

$$
\begin{aligned}
\theta(\xi, \rho) & =1+\sum_{n=1}^{\infty} \frac{e^{\beta_{n} \xi}}{\beta_{n}} \cdot \frac{f\left(\rho ; \beta_{n}, P \hat{e}\right)}{\frac{\partial f}{\partial p}\left(1 ; \beta_{n}, P \hat{e}\right)}, \xi>0 \\
& =-\sum_{n=1}^{\infty} \frac{e^{\alpha_{n} \xi}}{\alpha_{n}} \cdot \frac{f\left(\rho ; \alpha_{n}, P e ́\right)}{\frac{\partial f}{\partial p}\left(1 ; \alpha_{n}, P \hat{e}\right)}, \xi<0,
\end{aligned}
$$

where $0<\alpha_{1}<\alpha_{2} \ldots$ are the positive zeros of $f(1 ; p, P e ́)$ and $0>B_{1}>B_{2} \ldots$ are the negative zeros.

\section{Expansion of the solution function $f(p ; p, P e ́)$}

Assuming that the Péclet number is small, we solve equation (2.5) by expanding $f$ in powers of $P \vec{e}$.

$$
\text { Putting } f(\rho ; p, P e ́)=\sum_{n=0}^{\infty}(P e ́)^{n} f_{n}(\rho, p) \text {, we obtain }
$$

$$
\frac{d^{2} f_{0}}{d \rho^{2}}+\frac{1}{\rho} \frac{d f_{0}}{d \rho}+p^{2} f_{0}=0
$$

and

$$
\frac{d^{2} f_{n}}{d \rho^{2}}+\frac{1}{\rho} \frac{d f_{n}}{d \rho}+p^{2} f_{n}=2 p\left(1-p^{2}\right) f_{n-1} .
$$

Equation (3.1) has the obvious solution $f_{0}(p, p)=\mathrm{J}_{0}(p p)$, and substituting

$$
f_{n}(\rho, p)=\sum_{m=0}^{n} F_{n m^{J}+m}(p p)
$$

in equation (3.2) gives (after a tedious but otherwise straightforward calculation)

$$
F_{r m}=\frac{2^{m}}{3^{m} p^{n}} \sum_{s=0}^{m}\left(-\frac{1}{3}\right)^{s} \gamma_{m s} \rho^{2 m+3 s} \phi_{n-m-s}(\rho),
$$

where 


$$
\phi_{t}(\rho)=\left(\rho-\rho^{3} / 3\right)^{t} / t !
$$

and the $\gamma_{m s}$ satisfy the recurrence relation

$$
\gamma_{m s}=\left(\gamma_{m-1, s-1}+(2 s+1) \gamma_{m-1, s}+(s+1)^{2} \gamma_{m-1, s+1}\right) /(2 r+3 s)
$$

with $\gamma_{00}=1, \gamma_{m s}=0$ if $s>m, s<0$ or $m<0$.

It can be shown that

$$
\begin{aligned}
\gamma_{r r} & =\frac{1}{5^{r} r !} \\
\gamma_{r+1, r} & =\frac{1}{5^{r} r !}\left(\frac{2}{7} r+\frac{1}{2}\right) \\
\gamma_{r+2, r} & =\frac{1}{5^{r} r !}\left(\frac{2}{49} r^{2}+\frac{46}{245} r+\frac{7}{40}\right)
\end{aligned}
$$

and in general

$$
\gamma_{r+t, r}=\frac{1}{5^{r} r !}\left(a_{t t} r^{t}+\ldots+a_{t 0}\right)
$$

where $a_{t t}=\left(\frac{2}{7}\right)^{t} \cdot \frac{1}{t !}$. Hence

(3.7) $f(\rho ; p ; P e ́)=$

$$
\sum_{n=0}^{\infty}(P \hat{e})^{n} \sum_{m=0}^{n} \sum_{s=0}^{m}\left(-\frac{1}{3}\right)^{s} \gamma_{m s} \rho^{2 m+3 s} \phi_{n-m-s}(\rho) \frac{2^{m}}{3^{m} p^{m}} J_{n+m}(\rho p) .
$$

Rearranging the order of summation and using Lommel's expansion

$$
(z+h)^{-\frac{1}{2} \nu_{J}}\left((z+h)^{\frac{1}{2}}\right)=\sum_{m=0}^{\infty}\left(-\frac{1}{2} h\right)^{m} z^{-\frac{1}{2}(\nu+m)} J_{V+m}\left(z^{\frac{1}{2}}\right) / m !,
$$

we obtain finally

(3.9) $f(\rho ; p, P e ́)=\sum_{m=0}^{\infty} 2^{m} \sum_{s=0}^{m}(-1)^{s}\left(P e ́ \cdot p \cdot \rho^{4} / 3\right)^{m+s} \gamma_{m s} J_{2 m+s}(y) / y^{2 m+s}$, where

$$
y^{2}=p^{2} \rho^{2}-2 p P e ́\left(\rho^{2}-p^{4} / 3\right)
$$


Since $\left|\mathrm{J}_{n}(y) / y^{n}\right|$ has a maximum of $\left(\frac{1}{2}\right)^{n} / n !$ and is $O\left(y^{-n-\frac{1}{2}}\right)$ as $y \rightarrow \infty$, it is apparent that this series solution converges rapidly.

\section{Zeros of $f(1 ; p, P e ́)$}

When $\rho=1$, the expression (3.9) gives

$$
f(1 ; p, P \hat{e}) \sim J_{0}\left(\left(p-\frac{4}{3} p P e ́\right)^{\frac{1}{2}} .\right.
$$

Writing $c_{n}$ for the $n$-th positive zero of $J_{0}(x)$ we obtain immediately the asymptotic expression

$$
\alpha_{n}, \beta_{n} \sim \pm c_{n}\left(1+\left(2 P \hat{e} / 3 c_{n}\right)^{2}\right)^{\frac{1}{2}}+2 P \hat{e} / 3
$$

Alternately, substituting

$$
\alpha_{n}=\alpha_{n 0}+P e ́ \cdot \alpha_{n 1}+(P e \hat{e})^{2} \alpha_{n 2}+\cdots
$$

in (3.7) and equating powers of $P \hat{e}$, we obtain

$$
\begin{aligned}
\alpha_{n 0} & =c_{n} \\
\alpha_{n 1} & =\frac{3}{4}+\mathrm{J}_{3}\left(c_{n}\right) / 12 \mathrm{~J}_{1}\left(c_{n}\right) \\
& \sim \frac{2}{3} \text { as } n \rightarrow \infty,
\end{aligned}
$$

with similar but more complicated expressions for the following terms.

The equivalent expansion for $\beta_{n}$ is obviously

$$
\beta_{n}=-\alpha_{n 0}+P \hat{e} \alpha_{n 1}-(P \hat{e})^{2} \alpha_{n 2}+\ldots .
$$

These formulae have been used to calculate the values of $\alpha_{n 1}, \alpha_{n 2}, \alpha_{n 3}$ for $n=1,8$. It will be seen from these results that for small values of Pé, these first four terms in the expansion appear to be adequate.

Putting $\rho=1, y^{2}=p^{2}-\frac{4}{3} p P e ́$ in (3.9) and differentiating with respect to $p$, we obtain

(4.7) $\left.\frac{\partial f}{\partial p}\right|_{\rho=1}=$

$$
\sum_{m=0}^{\infty} 2^{m} \sum_{s=0}^{m}(-1)^{s} \gamma_{m, s}\left(\frac{p P e ́}{3}\right)^{m+s}\left[\frac{m+s}{p} \frac{J_{2 m+s}(y)}{y^{2 m+s}}-\left(p-\frac{2}{3} P e ́\right) \frac{J_{2 m+s+1}(y)}{y^{2 m+s+1}}\right] \text {, }
$$


from which the terms up to $m=5$ were used to calculate the coefficients in (2.8) for the first eight positive and negative eigenvalues

corresponding to $P e ́=\frac{1}{2}$ and $P e ́=1$.

Differentiating (3.9) with respect to $\rho$ we obtain

(4.8) $\left.\frac{\partial f}{\partial \rho}\right|_{\rho=1}=$

$$
p \sum_{m=0}^{\infty} 2^{m} \sum_{s=0}^{m}(-1)^{s} \gamma_{m, s}\left(\frac{p P \hat{e}}{3}\right]^{m+s}\left[\frac{4(m+s)}{p} \frac{\mathrm{J}_{2 m+s}(y)}{y^{2 m+s}}-\left(p-\frac{2}{3} P \hat{e}\right) \frac{\mathrm{J}_{2 m+s+1}(y)}{y^{2 m+s+1}}\right]
$$

from which $\left.\frac{\partial f}{\partial \rho}\right|_{\rho=1}$ can be calculated in a similar fashion. Since the dominant term in the expansion is given by $m=0, s=0$, we see that for large $p$,

$$
\left.\left.\frac{\partial f}{\partial \rho}\right|_{\rho=1} \sim p \frac{\partial f}{\partial p}\right|_{\rho=1}
$$

The eigenfunctions were calculated by rewriting equation (2.5) in the form

$$
f(\rho)=1-\int_{0}^{\rho} s \log (\rho / s)\left(p^{2}-2 p P e ́\left(1-s^{2}\right)\right) f(s) d s
$$

and integrating numerically.

The integral $\int_{0}^{l} \rho\left(1-\rho^{2}\right) f(\rho) d \rho$ was calculated simultaneously. This integral is required for evaluating the mean mixed temperature

$$
\theta_{M}=4 \int_{0}^{1} \rho\left(1-\rho^{2}\right) \theta(\xi, \rho) d \rho
$$

from which the Nusselt number

$$
M u=\frac{2 \frac{\partial \theta}{\partial \rho}}{\theta_{W}-\theta_{M}}
$$

is calculated, $\theta_{W}$ being the appropriate wall temperature.

The results of these computations are given in the tables and graphs 
below. The limiting Nusselt numbers as $\xi+\infty$ agree with those given by Pahor and Strnad [4].

In conclusion, it will be noted that the incoming fluid is significantly pre-heated and hence that the assumption that the temperature is constant for $x<0$ is not valid for low Péclet numbers.

Table 1. Coefficients in the expansion $\alpha_{n}=\alpha_{n 0}+P e \alpha_{n l}+\ldots$

\begin{tabular}{|c|c|c|c|}
\hline$n$ & $\alpha_{n 1}$ & $\alpha_{n 2}$ & $\alpha_{n 3}$ \\
\hline 1 & .78194 & .12112 & -.00405 \\
2 & .68855 & .04697 & .00172 \\
3 & .67557 & .03039 & .00072 \\
4 & .67146 & .02244 & .00038 \\
5 & .66966 & .01777 & .00024 \\
6 & .66871 & .01471 & .00016 \\
7 & .66815 & .01254 & .00011 \\
8 & .66779 & .01093 & .00009 \\
\hline
\end{tabular}


Table 2. Eigenvalues and coefficients for $P \hat{e}=1$

\begin{tabular}{|c|r|r|r|r|}
\hline$p$ & $\frac{\partial f}{\partial p}$ & $1 / p \frac{\partial f}{\partial p}$ & $\frac{\partial f}{\partial \rho}$ & $\int_{0}^{1} \rho\left(1-\rho^{2}\right) f(\rho) d \rho$ \\
\hline 3.29957 & -.41890 & -.58406 & -1.5136 & .16056 \\
6.27554 & .32531 & .49126 & 1.9986 & .00094 \\
9.36040 & -.26256 & -.40689 & -2.4425 & .00072 \\
12.4858 & .22592 & .35451 & 2.7886 & -.00032 \\
15.6186 & -.20229 & -.31651 & -3.1539 & .00016 \\
18.7546 & .18448 & .28903 & 3.4559 & -.00009 \\
21.8924 & -.17068 & -.26762 & -3.7337 & .00005 \\
25.0313 & .15958 & .25034 & 3.9923 & -.00003 \\
-1.74386 & .56511 & -1.01474 & -1.1299 & .14403 \\
-4.87694 & -.36041 & .56895 & 1.7732 & -.01433 \\
-8.00782 & .28227 & -.44240 & -2.2618 & .00289 \\
-11.1421 & -.24054 & .37312 & 2.6758 & -.00090 \\
-14.2788 & .21134 & -.33138 & -3.0163 & .00036 \\
-17.4169 & -.19132 & .30011 & 3.3307 & -.00018 \\
-20.5559 & .17608 & -.27629 & -3.6182 & .00010 \\
-23.6955 & -.16398 & .25736 & 3.8845 & -.00006 \\
& & & & \\
\hline
\end{tabular}


Table 3. Eigenvalues and coefficients for $P e ́=.5$

\begin{tabular}{|c|c|c|c|c|}
\hline$p$ & $\frac{\partial f}{\partial p}$ & $1 / p \frac{\partial f}{\partial p}$ & $\frac{\partial f}{\partial \rho}$ & $\int_{0}^{1} \rho\left(1-\rho^{2}\right) f(\rho) d \rho$ \\
\hline 2.82530 & -.51374 & -.68895 & -1.3550 & .15392 \\
5.87631 & .33201 & .51256 & 1.9368 & -.00398 \\
8.99920 & -.26675 & -.41658 & -2.3951 & .00115 \\
12.1329 & .22942 & .35926 & 2.7806 & -.00043 \\
15.2702 & -.20435 & -.32047 & -3.1186 & .00020 \\
18.4091 & .18606 & .29195 & 3.4240 & -.00011 \\
21.5489 & -.17195 & -.26989 & -3.7043 & .00006 \\
24.6891 & .16062 & .25217 & 3.9649 & -.00004 \\
-2.04437 & .56319 & -.91227 & -1.1773 & .14616 \\
-5.18733 & -.34979 & .55113 & 1.8236 & -.01151 \\
-8.32345 & .27664 & -.43430 & -2.3046 & .00227 \\
-11.4614 & -.23496 & .37134 & 2.6535 & -.00072 \\
-14.6005 & .20888 & -.32790 & -3.0498 & .00030 \\
-17.7404 & -.18948 & .29749 & 3.3614 & -.00015 \\
-20.8807 & .17464 & -.27422 & -3.6465 & .00008 \\
-24.0213 & -.16282 & .25568 & 3.9110 & -.00005 \\
& & & & \\
\hline
\end{tabular}


Table 4. Mean mixed temperatures and Nusselt numbers

\begin{tabular}{|r|c|c|c|r|r|r|r|}
\hline \multicolumn{1}{|c|}{$P \hat{e}=1$} & \multicolumn{5}{c|}{$P \hat{e}=.5$} \\
\hline$\xi$ & $\theta_{M}$ & $\frac{\partial \theta}{\partial \rho}$ & $M u$ & $\xi$ & $\theta_{M}$ & $\frac{\partial \theta}{\partial \rho}$ & $N u$ \\
\hline-10.0 & .0000 & -.0000 & 4.7135 & -10.0 & .0000 & -.0000 & 4.4016 \\
-1.0 & .0138 & -.0346 & 4.9995 & -1.0 & .0252 & -.0583 & 4.6291 \\
-.5 & .0720 & -.2244 & 6.2358 & -.5 & .1037 & -.2939 & 5.6662 \\
-.1 & .2694 & -2.3903 & 17.7484 & -.1 & .3254 & -2.5342 & 15.5779 \\
0 & .3754 & $\infty$ & $\infty$ & 0 & .4355 & $\infty$ & $\infty$ \\
.1 & .4860 & +2.9958 & 11.6569 & .1 & .5480 & 2.8386 & 12.5595 \\
.5 & .7526 & +.5905 & 4.7791 & .5 & .8061 & .4812 & 4.9643 \\
1.0 & .8995 & .2085 & 4.0697 & 1.0 & .9308 & .1449 & 4.1888 \\
10.0 & 1.0000 & .0000 & 3.9224 & 10.0 & 1.0000 & .0000 & 4.0027 \\
\hline
\end{tabular}
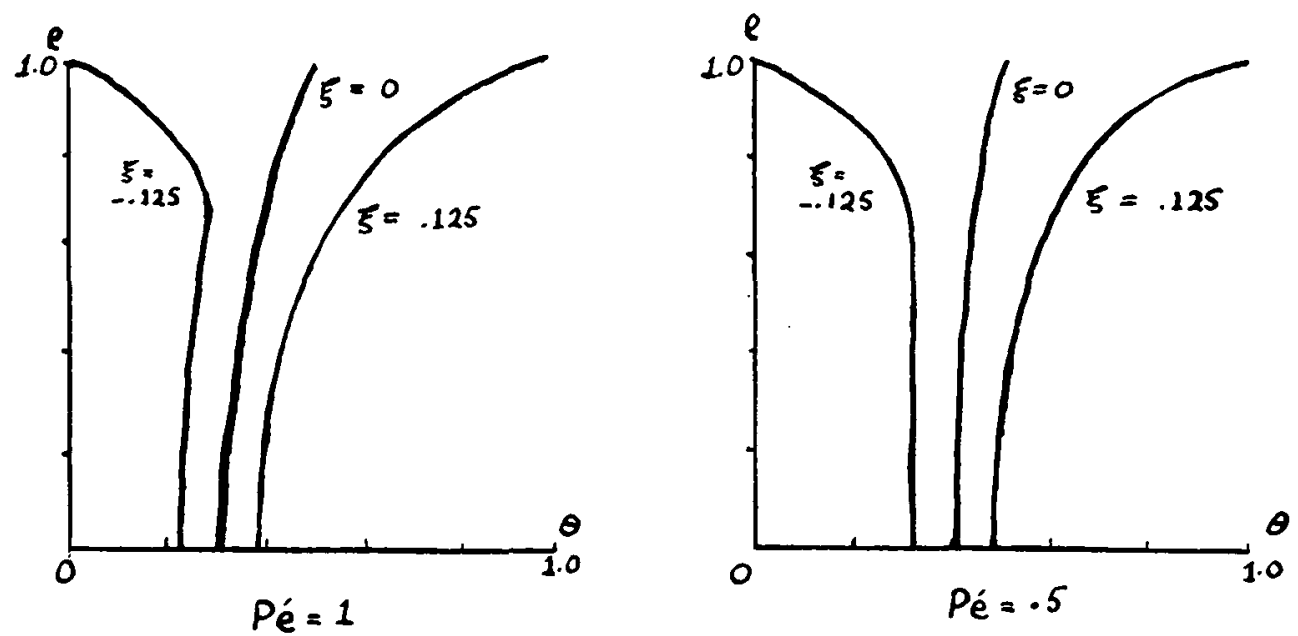

Figure 1. Thermal profiles near the origin. The profiles for $\xi=0$ are the means of the limits $\xi \rightarrow 0+$ and $\xi \rightarrow 0-$. 


\section{References}

[1] M. Abramowitz, W.F. Cahill and C. Wade, Jr, "Heat transfer in laminar flow through a tube", J. Res. Nat. Bur. Standards 62 (1959), 101-105.

[2] H.C. Agrawal, "Heat transfer in laminar flow between parallel plates at small Péclét numbers", Appl. Sci. Res. A9 (1960), $177-189$.

[3] Knox Millsaps and Karl Pohlhausen, "Heat transfer to Hagen-Poiseuille flows", Proc. Conf. Differential Equations, Univ. Maryland, MaryLand, 1955, 271-294 (University of Maryland Bookstore, College Park, Maryland, 1956).

[4] Sergej Pahor and Janez Strnad, "Heat transfer from laminar flow through cylindrical tubes", 2. angew. Math. Phys. 12 (1961), 80-81.

[5] S.N. Singh, "Heat transfer by laminar flow in a cylindrical tube", App 2. Sci. Res. A7 (1958), 325-340.

Department of Mathematics,

University of Queensland,

St Lucia,

Queensland. 